\title{
Response and Modeling of Cantilever Retaining Walls Subjected to Seismic Motions
}

\author{
Russell A. Green* \\ Department of Civil and Environmental Engineering, University of Michigan, 2372 G.G. Brown, \\ Ann Arbor, MI, 48109-2125, USA \\ C. Guney Olgun \\ Department of Civil and Environmental Engineering, Virginia Polytechnic Institute and State University, \\ Blacksburg, VA, 24061, USA
}

$\&$

Wanda I. Cameron

Department of Civil and Environmental Engineering, University of Michigan, 2372 G.G. Brown, Ann Arbor, MI, 48109-2125, USA

\begin{abstract}
A series of nonlinear, explicit finite difference analyses were performed to determine the dynamic response of a cantilever retaining wall subjected to earthquake motions. This article outlines the calibration and validation of the numerical model used in the analyses and comparisons are presented between the results from the finite difference analyses and results from simplified techniques for computing dynamic earth pressures and permanent wall displacement (i.e., Mononobe-Okabe and Newmark sliding block methods). It was found that at very low levels of acceleration, the induced pressures were in general agreement with those predicted by the MononobeOkabe method. However, as the accelerations increased to those expected in regions of moderate seismicity, the induced pressures are larger than those predicted by the Mononobe-Okabe method. This deviation is attributed to the flexibility of the retaining wall system and to the observation that the driving soil wedge does not respond monolithically, but rather responds as several wedges. Ad-

\footnotetext{
*To whom correspondence should be addressed. E-mail: rugreen@
} umich.edu.
\end{abstract}

ditionally, it was found that the critical load case for the structural design of the wall differed from that for the global stability of the wall, contrary to the common assumption made in practice that the two load cases are the same.

\section{INTRODUCTION}

Presented herein is an overview of a numerical model and analyses performed to determine the trends in the dynamic response of cantilever retaining walls subjected to earthquake motions, where the walls are allowed to flex, rotate, and slide. Significant emphasis is placed on identifying trends in the wall response and on calibrating and validating the soil-wall system model used in this study, as opposed to the development and implementation of advanced component level models (e.g., soil and wall constitutive models). In this vein, the componentlevel models used in this study are available as options in many commercial finite element and finite difference software packages. However, the validation procedure 
used is readily extendable system models that incorporate advanced, user-developed component-level models.

True validation of any system model/analysis approach requires good agreement between predicted and actual field performance data, not only at the end of shaking but throughout the entire duration of shaking. Unfortunately, such detailed and well-documented field performance data requisite for proper validation of seismic analyses of cantilever retaining walls (and for almost any type of structure) do not exist. Consequently, the validation approach adopted by the authors was to perform the analyses using system models that have increasing complexity and to evaluate the trends in the response by comparing them to results from simple analytical models (e.g., Okabe, 1924; Mononobe and Matsuo, 1929; Newmark, 1965). When the response results differed either in trend or significantly in amplitude, efforts were spent to determine whether the differences were due to inherent limitations of the simple models or whether the more complex system model was flawed. This assessment was facilitated by performing limited parametric studies using both the complex and simple models and also by examining trends identified in published results from the physical model tests (i.e., shaking table and centrifuge tests). The computer code, Fast Lagrangian Analysis of Continua (FLAC) (Itasca, 2000) was used to perform the numerical analyses (i.e., nonlinear, explicit finite difference analyses). The analyses consisted of the incremental construction of the wall and placement of the backfill, followed by dynamic response analyses, wherein the soil was modeled as elasto-plastic with a Mohr-Coulomb failure criterion.

Brief mention needs to be made of the significant studies that have been previously published for estimating the seismically induced forces on retaining walls and/or to estimate the seismically induced displacements of retaining walls (e.g., Wood, 1973; Richards and Elms, 1979; Nadim and Whitman, 1983; Veletsos and Younan, 1994; Wu and Finn, 1999; Ostadan, 2005) and how the study presented herein differs from them. Most previous studies have focused on gravity retaining wall systems or nonyielding walls. Cantilever walls, which are the focus of this study, differ from gravity walls and nonyielding walls, in that structural stability as well as global stability need to be considered, with the critical load case for the two being different as concluded from this study. Also, the more complex numerical analyses are used to highlight the limitations of the simple analytical approaches.

Regarding the organization of the remaining parts of this article, first a description of the wall-soil system is presented, followed by an overview of the finite difference model used in the study. Next, a discussion of how the ground motions were specified, how the wall and soil model parameters were determined, and how the wall-soil interface was modeled are presented. Comparisons of the finite difference results are made with results from simplified analysis techniques for determining dynamic earth pressures (i.e., Mononobe-Okabe approach (Okabe, 1924; Mononobe and Matsuo, 1929) and permanent displacement of the wall (i.e., Newmark sliding block approach (Newmark, 1965)). Subsequent to these comparisons, additional observations made from the finite difference analyses are discussed.

\section{NUMERICAL MODEL}

\subsection{Description of wall-soil system}

The retaining wall analyzed was approximately $6.1 \mathrm{~m}$ in height, retaining medium-dense, cohesionless, compacted fill (total unit weight: $\gamma_{t}=19.6 \mathrm{kN} / \mathrm{m}^{3}$; effective angle of internal friction: $\phi^{\prime}=35^{\circ}$ ). Underlying the wall/backfill was approximately $62.5 \mathrm{~m}$ of naturally deposited dense cohesionless soil $\left(\gamma_{t}=19.6 \mathrm{kN} / \mathrm{m}^{3} ; \phi^{\prime}=\right.$ $40^{\circ}$ ). The small strain shear wave velocity profile of the soil deposit is shown in Figure 1. The groundwater table was well below the base of the wall and was not considered in the analyses. The geometry and the properties

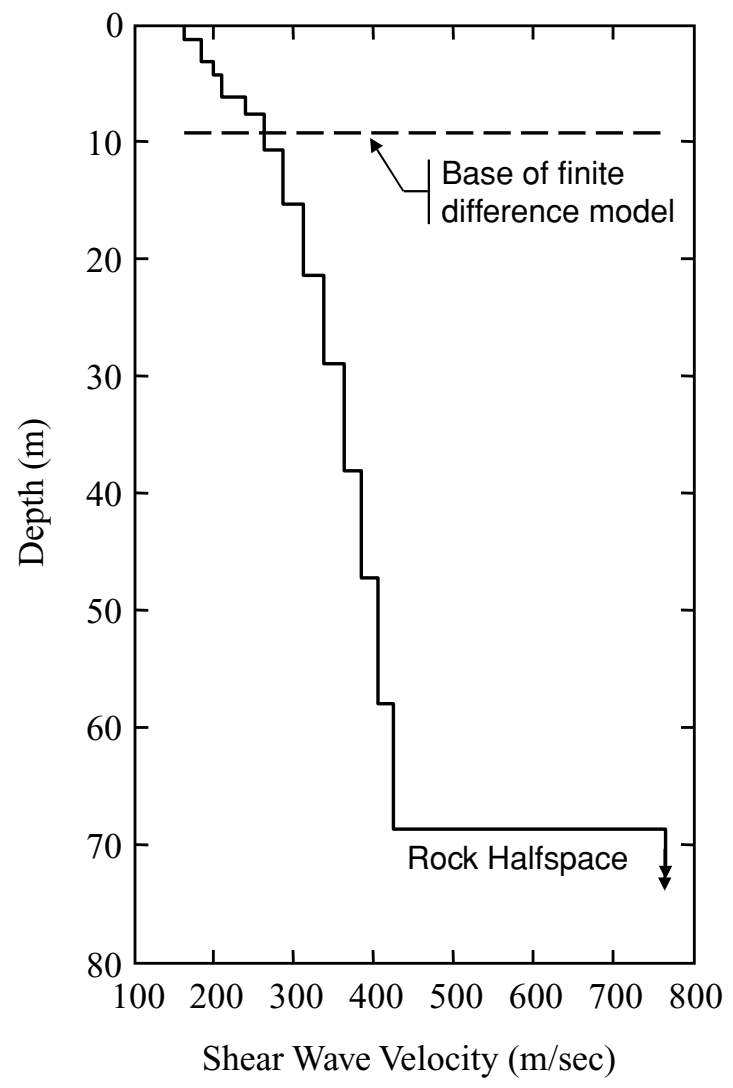

Fig. 1. Small strain shear wave velocity profile analyzed. 


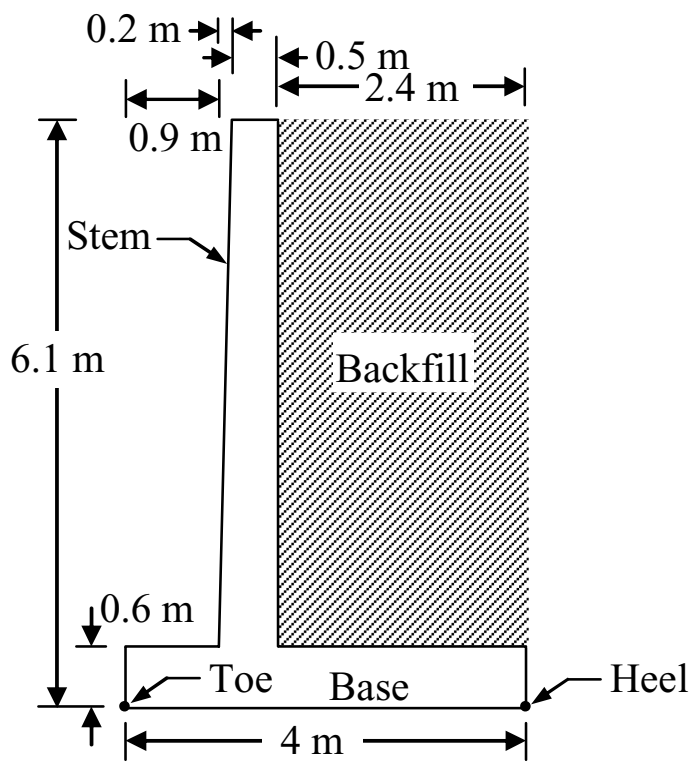

Fig. 2. Dimensions of the structural block of the wall-soil system analyzed, wherein the term "structural block" refers to all that is shown above.

of the wall-soil system were specified by the U.S. Army Corps of Engineers (Ebeling and Filz, 2001) and are considered to be "representative" of many medium-height walls in the United States.

The geometry and structural detailing of the wall were determined following the U.S. Army Corps of Engineers static design procedures (Headquarters, U.S. Army Corps of Headquarters, US Army Corps of Engineers, 1989, 1992), with the dimensions of the structural block (i.e., wall and contained backfill) depicted in Figure 2. The properties of the concrete and reinforcing steel used in the wall design are as follows: unit weight of concrete: $\gamma_{c}=23.6 \mathrm{kN} / \mathrm{m}^{3}$; compressive strength of concrete: $f_{c}^{\prime}=$ $27.6 \mathrm{MPa}$; and yield strength of reinforcement: $f_{y}=413.4$ $\mathrm{MPa}$. Additional details about the wall design and soil profile are given in Green and Ebeling (2002).

\subsection{Overview of finite difference model}

The finite difference model consisted of the upper 9.1 $\mathrm{m}$ of the wall-soil system, comprising the wall/backfill and approximately $3 \mathrm{~m}$ of the underlying natural deposit (foundation soil). Laterally, the model was approximately $22.9 \mathrm{~m}$ wide, to include approximately $7.6 \mathrm{~m}$ of the foundation soil in front of the wall and approximately $15.3 \mathrm{~m}$ of the backfill/foundation soil behind the wall (Figure 3).

An elasto-plastic constitutive model, in conjunction with Mohr-Coulomb yield condition, was used to model the soil. The dilatancy angle for the soil was assumed

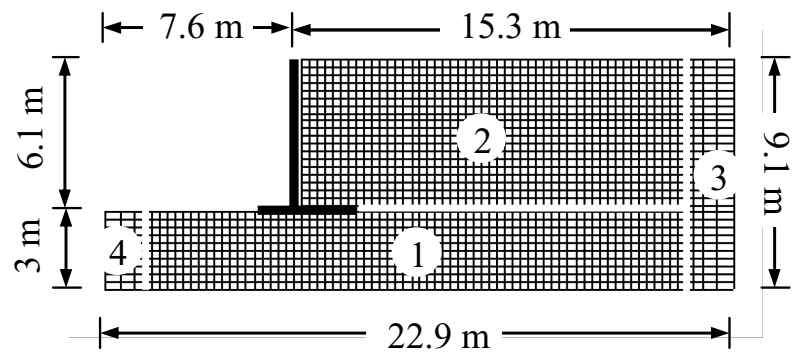

Fig. 3. Annotated finite difference mesh of the wall-soil system.

equal to zero (i.e., nonassociative flow rule). Elastic beam elements were used to model the concrete retaining wall, with the wall/backfill being "numerically constructed" similar to the way an actual wall would be constructed. The backfill was placed in $0.61 \mathrm{~m}$ lifts, for a total of 10 lifts, with the model being brought to static equilibrium after the placement of each lift. Such placement allowed realistic earth pressures to develop as the wall deformed and moved in response to the placement of each lift. The constructed retaining wall-soil model is shown in Figure 3.

The model consists of four subgrids, labeled one through four in Figure 3. The separation of the foundation soil and backfill into subgrids one and two was required because a portion of the base of the retaining wall was inserted into the soil/backfill. Subgrid three was included so that free-field boundary conditions could be specified along the lateral edges of the model (free-field boundary conditions cannot be specified across the interface of two subgrids). Subgrid four was included for symmetry, but its inclusion was not necessary. The subgrids were "attached" at the soil-to-soil interfaces, as depicted by white lines in Figure 3, and interface elements were used at the wall-soil interfaces.

The following subsections outline how the ground motions were specified and the procedures used to determine the various soil and wall model parameters.

\subsection{Specification of input motions}

In FLAC, the dynamic motions can be specified as acceleration, velocity, stress, or force time histories at the exterior boundaries or as interior excitations. A parametric study was performed to determine the best way to specify the ground motions for earthquake analyses. The parametric study involved performing a series of one-dimensional (1-D) site response analyses using consistently generated acceleration, velocity, and stress time histories. Generally, earthquake ground motions are not defined in terms of force time histories and, therefore, were not considered in the parametric study. The use of 
stress time histories has the benefit of allowing the time history to be specified at energy absorbing (or "quiet boundaries"), thus simulating radiation damping.

Using free-field acceleration time histories recorded at the surface of USGS site class B profiles, 1-D site response analyses were performed using a modified version of SHAKE91 (Idriss and Sun, 1992). The analyses were performed on a $68.6 \mathrm{~m}, 5 \%$ damped, nondegrading profile, wherein the acceleration time histories were specified as outcrop motions. Interlayer acceleration and stress time histories were computed at the profile surface and at depths of 7.6, 10.7, 15.2, and $68.6 \mathrm{~m}$ (i.e., bedrock). Interlayer velocity time histories were computed by integrating the interlayer acceleration time histories using the trapezoidal rule.

The interlayer acceleration, velocity, and stress time histories computed using SHAKE were used as base motions in a series of finite difference site response analyses, in which the acceleration time histories at the surface of the finite difference profiles were computed. The profiles used in the finite difference analyses were comparable to the SHAKE profiles down to the depths corresponding to the interlayer motions. An elastic constitutive relation, with 5\% Rayleigh damping, was used to model the soil layers in the finite difference profiles. The central frequency of the damping relationship was set to the fundamental frequencies of the respective finite difference profiles.

Fourier amplitude spectra (FAS) and 5\% damped, pseudo-acceleration response spectra (PSA) were computed from the acceleration time histories of the surface motions of the SHAKE and finite difference profiles. Error analyses were performed on the spectra corresponding to the different profiles and different types of specified input motions. In the error analyses, the spectra for the SHAKE motions were used as the "correct" motions. The word "correct" does not imply that SHAKE precisely models the behavior of an actual soil profile subjected to earthquake motions. Rather, SHAKE gives the analytically correct motion for a visco-elastic profile with constant damping applied to all frequencies of motion. On the other hand, the finite difference models used in this study give numerical approximations of the correct analytical solution. The errors in the finite difference spectral values were computed for a spectrum of frequencies using the following expressions:

$$
\begin{aligned}
& \text { Error }_{P S A}=\frac{P S A_{F L A C}-P S A_{\mathrm{SHAKE}}}{P S A_{\mathrm{SHAKE}}} \\
& \text { Error }_{F A S}=\frac{F A S_{F L A C}-F A S_{\mathrm{SHAKE}}}{F A S_{\mathrm{SHAKE}}}
\end{aligned}
$$

From the results of the parametric study, it was determined that the specification of the input motion in FLAC in terms of stress time histories gives the least accurate results, wherein the stress time histories were applied at a "quiet boundary" along the base of the FLAC model. The errors corresponding to specifying the motions in terms of acceleration and velocity time histories were essentially identical and considerably less than those associated with the stress time histories.

\subsection{Development of input motions for wall analyses}

As stated previously, the finite difference model of the soil-wall system consisted of only the upper $9.1 \mathrm{~m}$ of a $68.6 \mathrm{~m}$ profile. To account for the influence of the soil profile below $9.1 \mathrm{~m}$ on the ground motions, the entire $68.6 \mathrm{~m}$ profile, without the retaining wall, was modeled using a modified version of SHAKE91. The interlayer motions at the depth corresponding to the base of the finite difference model (i.e., $9.1 \mathrm{~m}$ ) were computed. The input ground motions used in the SHAKE analyses were the same motions used in the parametric study discussed above. The motions were specified as rock outcrop motions at the base of the $68.6 \mathrm{~m}$ soil column.

The small strain fundamental frequency of the retaining wall-soil system in the finite difference model was estimated to be approximately $6 \mathrm{~Hz}$. At larger strains, the fundamental frequency of the system will be less than the small strain value. To ensure proper excitation of the wall, the cutoff frequency in the SHAKE analyses were set at $15 \mathrm{~Hz}$. This value was selected considering both the fundamental frequency of the wall-soil system and the fact that the input motions had little energy at higher frequencies. The interlayer motions (at $9.1 \mathrm{~m}$ depth) computed using SHAKE were specified as acceleration time histories along the base of the finite difference model.

\subsection{Model parameters for soil}

The soil was modeled using an elastic-perfectly plastic constitutive relation, with the Mohr-Coulomb envelope defining the yield criterion. The plastic flow rule was assumed to be nonassociative, as is appropriate for cohesionless soils. Five parameters are required for this model to be fully described: effective internal friction angle $\left(\phi^{\prime}\right)$; dilatancy angle $(\zeta)$; mass density $(\rho)$; small strain shear modulus $\left(G_{\max }\right)$; and bulk modulus $(K)$. Both $\phi^{\prime}$ and $\rho$ are familiar to geotechnical engineers, where $\rho$ is the total unit weight of the soil $\left(\gamma_{t}\right)$ divided by the coefficient of acceleration due to gravity $(g)$, that is, $\rho=\gamma_{t} / g$. As stated previously, $\phi^{\prime}$ for the backfill and foundation soil were $35^{\circ}$ and $40^{\circ}$, respectively. These values are consistent with medium-dense compacted fill and dense natural deposits.

$\zeta$ is the angle of dilation that can vary from 0 to $\phi^{\prime}$ depending on the form of the plastic potential function used in the flow rule: 


$$
\dot{\varepsilon}_{i j}=\dot{\lambda} \frac{\partial g\left(\sigma_{i j}\right)}{\partial \sigma_{i j}}
$$

where $\dot{\varepsilon}_{i j}$ and $\sigma_{i j}$ are the strain rate and stress tensors, respectively; $\mathrm{g}\left(\sigma_{i j}\right)$ is the plastic potential function; and $\dot{\lambda}$ is a nonnegative multiplier for an elastic-perfectly plastic material (not a material property). When $\zeta=\phi^{\prime}$, the plastic potential takes a form identical to the yield criterion (i.e., $g\left(\sigma_{i j}\right)=f\left(\sigma_{i j}\right)$, where $f\left(\sigma_{i j}\right)=$ the yield criterion); hence, the flow rule becomes associative. For granular soils falling on the dense side of the critical state line and sheared drained, as is the case for the backfill and foundation soil, $\zeta<\phi^{\prime}$. For the type and state of the backfill and foundation soil being analyzed, dilation angles of $5^{\circ}$ to $10^{\circ}$ would be representative. However, it is the authors' experience that when soil is not significantly restrained, such as for slopes and retaining walls that can slide, the assumed dilatancy angle has little influence on the resulting system response. Consequently, the soil was assumed to be incompressible (i.e., $\zeta=0$ ).

Several correlations exist that relate $G_{\max }$ to other soil parameters. However, the most direct relation is between $G_{\max }$ and small strain shear wave velocity $\left(v_{s}\right)$ :

$$
G_{\max }=\rho \cdot v_{s}^{2}
$$

where $v_{s}$ may be determined by various types of site characterization techniques, such as cross hole or spectral analysis of surface waves (SASW) studies (e.g., Stokoe et al., 1994; Woods, 1994).

Values for $K$ can be determined from $G_{\max }$ and Poisson's ratio $(v)$ using the following relation:

$$
K=\frac{2 \cdot G_{\max } \cdot(1+v)}{3 \cdot(1-2 \cdot v)}
$$

in which $v$ may be estimated using the following expression:

$$
v=\frac{1-\sin \left(\phi^{\prime}\right)}{2-\sin \left(\phi^{\prime}\right)}
$$

which was derived from the theory of elasticity (e.g., Terzaghi, 1943), in conjunction with the correlation relating $K_{o}$ and $\phi^{\prime}$ proposed by Jaky (1944), that is, $K_{o}=1-$ $\sin \left(\phi^{\prime}\right)$. Using the above expression, $v$ was determined to be 0.26 and 0.3 for the foundation soil and backfill, respectively.

\subsection{Model parameters for wall}

The concrete wall was divided into five segments having constant parameters, as illustrated in Figure 4, with each segment consisting of several $0.3 \mathrm{~m}$ elastic beam elements. Four parameters were required to define the mechanical properties of the elastic beam elements: cross-sectional area $\left(A_{g}\right)$; mass density $(\rho)$; elastic mod-

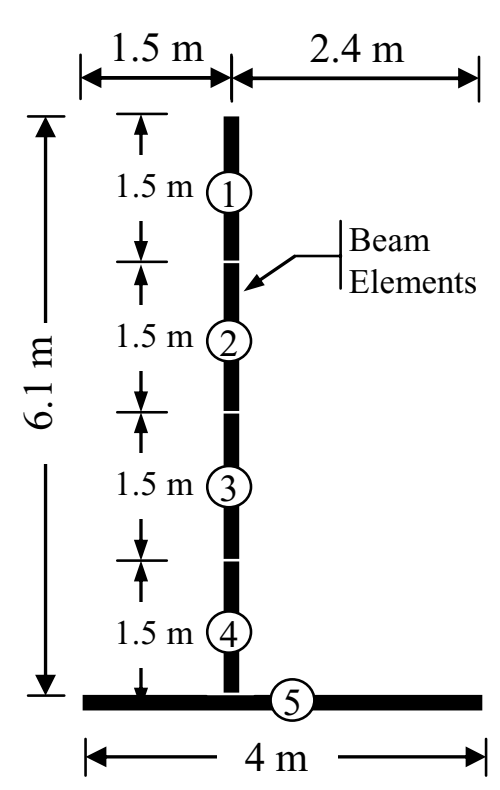

Fig. 4. Numerical model of retaining wall using elastic beam elements.

ulus $\left(E_{c}\right)$; and second moment of area $(I)$, commonly referred to as moment of inertia.

The basis for subdividing the wall into five segments was the variation of the mechanical properties along the height of the wall. A wall having a greater taper or largely varying steel reinforcement along the length of the stem or base would likely require more segments. For each of the segments, $A_{g}$ and $\rho$ were readily determined from the wall geometry and the unit weight of the concrete (i.e., $\left.23.6 \mathrm{kN} / \mathrm{m}^{3}\right)$. $E_{c}$ was computed using the following expression (e.g., MacGregor, 1992):

$$
E_{c}=57,000 \cdot \sqrt{f_{c}^{\prime}}
$$

In this expression, $f_{c}^{\prime}$ is the compressive strength of the concrete (e.g., 4,000 psi for the wall being modeled), and both $E_{c}$ and $f_{c}^{\prime}$ are in psi. Because the structure is continuous in the direction perpendicular to the analysis plane, $E_{c}$ that was computed using Equation (6) needed to be modified to account for plane-strain conditions. This modification was done using the following expression (Itasca, 2000):

$$
E_{\text {c plane strain }}=\frac{E_{c}}{\left(1-v^{2}\right)}
$$

where 0.2 was assumed for Poisson's ratio for concrete.

$I$ is a function of the geometry of the segments, the amount and location of the reinforcing steel, and the amount of cracking in the concrete, where the latter in turn depends on the static and dynamic load imposed on the member. In dynamic analyses, it is difficult to state a priori whether the use of sectional properties 


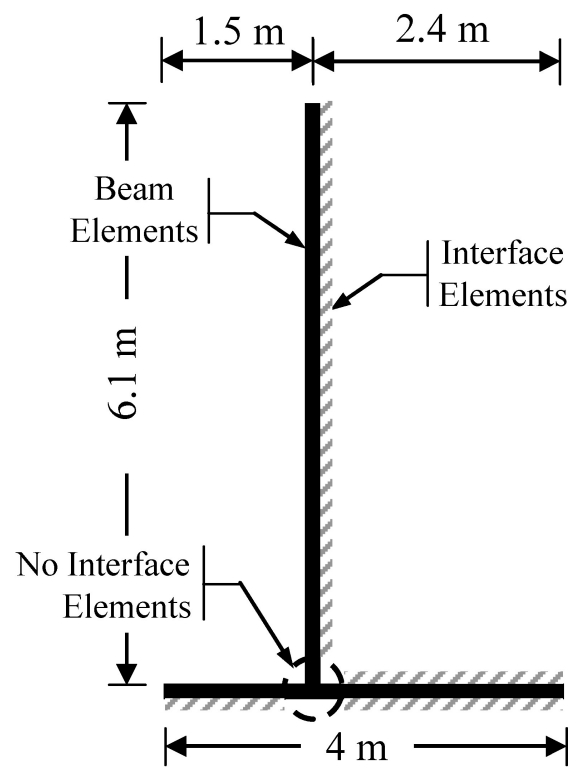

Fig. 5. Location of interface elements in the finite difference model.

corresponding to uncracked, fully cracked, or some intermediate level of cracking will result in the largest demand on the structure. However, $I=0.4 \times I_{\text {uncracked }}$ was used as a reasonable estimate for the sectional properties (Paulay and Priestley, 1992).

\subsection{Model parameters for wall-soil interface}

Interface elements were used to model the interaction between the concrete retaining wall and the soil. However, the finite difference code does not allow interface elements to be used at the intersection of branching structures (e.g., the intersection of the stem and base of the cantilever wall). Several approaches were attempted by the authors to circumvent this limitation in finite difference code, with the simplest and best approach, as found by the authors, illustrated in Figure 5. As shown in this figure, three very short beam elements, oriented in the direction of the stem, toe side of the base, and heel side of the base, were used to model the base-stem intersection. No interface elements were used on these three short beam elements. However, interface elements were used along the other contact surfaces between the soil and wall, as depicted by the hatched areas in Figure 5.

A schematic of the interface element is presented in Figure 6. As may be observed from this figure, the interface element has four parameters: $S=$ slider representing shear strength; $T=$ tensile strength; $k_{n}=$ normal stiffness; and $k_{s}=$ shear stiffness. The element allows permanent separation and slip of the soil and the structure,

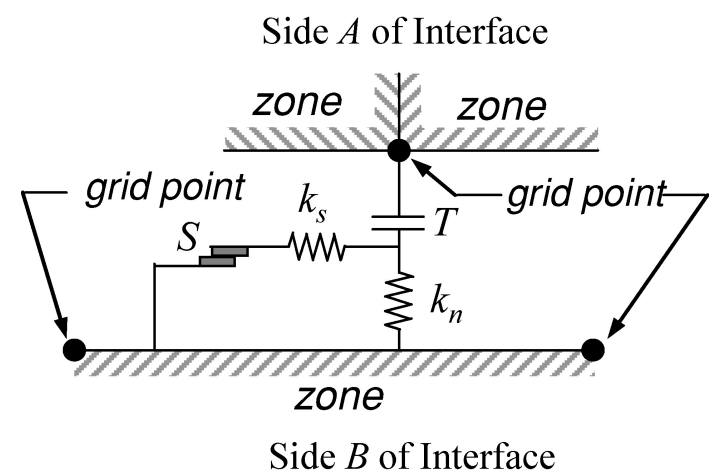

Fig. 6. Schematic of the finite difference interface element (adapted from Itasca, 2000).

as controlled by the parameters $T$ and $S$, respectively. For the cohesionless soil being modeled, $T=0$ (i.e., cohesionless soil), whereas $S$ was specified as a function of the interface friction angle $(\delta)$. For medium-dense sand against concrete, $\delta=31^{\circ}$ (Gomez et al., 2000a).

As a rule-of-thumb, $k_{n}$ should be set to 10 times the equivalent stiffness of the stiffest neighboring zone (Itasca, 2000):

$$
k_{n} \approx 10 \cdot \max \left[\frac{K+\frac{4}{3} \cdot G_{\max }}{\Delta z_{\min }}\right]
$$

In Equation (8), $K$ and $G_{\max }$ are the bulk and small strain shear moduli, respectively, and $\Delta z_{\min }$ is the smallest width of a zone in the normal direction of the interfacing surface. The max[] notation indicates that the maximum value over all zones adjacent to the interface be used. Arbitrarily large values for $k_{n}$, as commonly used in implicit finite element analyses, should not be used, as this results in unnecessarily small time steps, and therefore unnecessarily long computational times (Itasca, 2000).

The determination of the $k_{s}$ required considerably more effort than the determination of the other interface element parameters. In shear, the interface element essentially is an elasto-plastic model, with an elastic stiffness of $k_{s}$ and yield strength $S$. The $k_{s}$ values were selected such that the resulting elasto-plastic model gave an approximate fit of the hyperbolic-type interface model proposed by Gomez et al. (2000a, b). A comparison of the two models for initial loading (i.e., construction of the wall) is shown in Figure 7.

The procedure used to determine $k_{s}$ values for initial loading is outlined below. The reader is referred to Gomez et al. (2000a, b) for more details concerning their proposed hyperbolic-type model. 


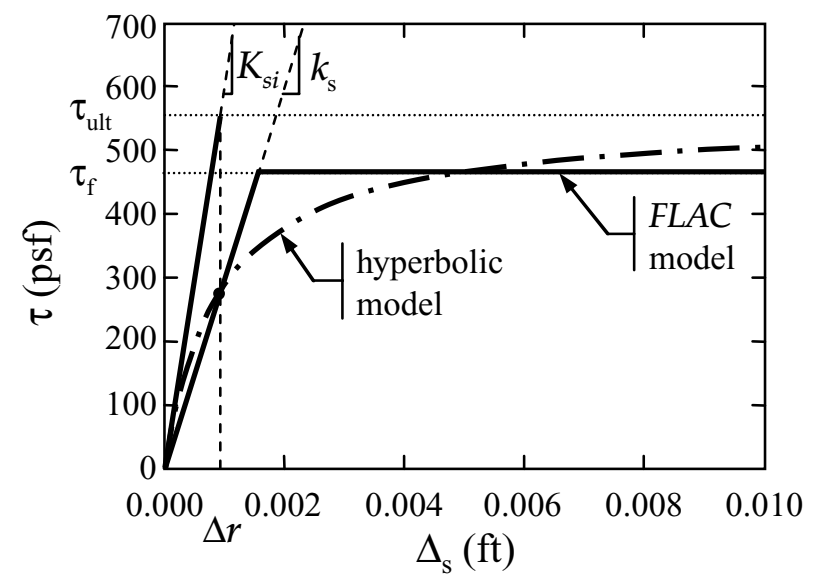

Fig. 7. Calibration of the $F L A C$ interface model to the hyperbolic-type model proposed by Gomez et al. (2000a,b).

1. Compute $\Delta r$ using the following expression.

$$
\Delta r=\frac{\tau_{f}}{R_{f j} \cdot K_{s i}}
$$

where,

$$
\begin{gathered}
\tau_{f}=\sigma_{n} \cdot \tan (\delta) \\
K_{s i}=K_{I} \cdot \gamma_{w} \cdot\left(\frac{\sigma_{n}}{P_{a}}\right)^{n_{j}}
\end{gathered}
$$

where $K_{s i}$ is the dimensionless initial shear stiffness of the interface; $\sigma_{n}$ is normal stress acting on the interface (determined iteratively by first assuming a small value for $k_{s}$ and then constructing the wall); $\delta$ is interface friction angle $=31^{\circ} ; R_{f j}$ is the failure ratio $=0.84 ; K_{I}$ is dimensionless interface stiffness number for initial loading $=21,000 ; n_{j}$ is dimensionless stiffness exponent $=0.8 ; \gamma_{w}$ is unit weight of water in consistent units as $\Delta r$; and $P_{a}$ is the atmospheric pressure in the same units as $\sigma_{n}$. The values for $R_{f j}, K_{I}, n_{j}$, and $\delta$ were obtained from Gomez et al. (2000b).

2. $k_{s}$ was computed using the following expression:

$$
k_{s}=\frac{1}{\frac{1}{K_{I} \cdot \gamma_{w} \cdot\left(\frac{\sigma_{n}}{P_{a}}\right)^{n_{j}}}+\frac{R_{f j} \cdot \Delta r}{\sigma_{n} \cdot \tan (\delta)}}
$$

The above computed $k_{s}$ values were used only for the initial construction of the wall. The $k_{s}$ values were changed after the construction of the wall and prior to the application of the earthquake loading to values consistent with the Gomez-Filz-Ebeling Version I load/unload/reload extended hyperbolic interface model (Gomez et al., 2000a). The procedure used to compute $k_{s}$ for the cyclic loading is outlined below. Again, the reader is referred to the cited report for more details concerning this model.

$$
k_{s}=K_{u r j} \cdot \gamma_{w} \cdot\left(\frac{\sigma_{n}}{P_{a}}\right)^{n_{j}}
$$

where,

$$
\begin{gathered}
K_{u r j}=C_{k} \cdot K_{I} \\
C_{k}=0.5 \cdot\left(1+R_{f j}\right)^{2}
\end{gathered}
$$

where $K_{u r j}$ is the unload-reload stiffness number for interfaces and $C_{k}$ is interface stiffness ratio.

Using the above expressions, the interface stiffnesses were computed for the interface elements identified in Figure 5. Although the $k_{s}$ for unloadreload were higher than the corresponding values for initial loading (i.e., Equation (11a) vs. Equation (10)), the values for $k_{n}$ were the same for both initial loading and unload-reload.

\subsection{Dimensions of finite difference zones}

Proper dimensioning of the finite difference zones is required to avoid numerical distortion of propagating ground motions, in addition to accurate computation of model response. Itasca (2000) recommends that the length of the element $(\Delta l)$ be smaller than one-tenth to one-eighth of the wavelength $(\lambda)$ associated with the highest frequency $\left(f_{\max }\right)$ component of the input motion. The basis for this recommendation is a study by Kuhlemeyer and Lysmer (1973). Interestingly, Lysmer et al. (1975) recommend $\Delta l$ to be smaller than one-fifth the $\lambda$ associated with $f_{\max }$, and also refer to Kuhlemeyer and Lysmer (1973) as the basis for the recommendation, that is:

$$
\begin{gathered}
\text { Itasca (2000): } \quad \Delta l \leq \frac{\lambda}{10} \\
\text { Lysmer et al. (1975): } \quad \Delta l \leq \frac{\lambda}{5}
\end{gathered}
$$

where $\lambda$ is related to the shear wave velocity of the soil $\left(v_{s}\right)$ and the frequency $(f)$ of the propagating wave by the following relation:

$$
\lambda=\frac{v_{s}}{f}
$$

Assuming that the response of the retaining wall will be dominated by shear waves, substituting Equation (12) into Equation (11a) gives:

$$
\Delta l \leq \frac{v_{s}}{10 \cdot f_{\max }}
$$

or

$$
f_{\max } \leq \frac{v_{s}}{10 \cdot \Delta l}
$$


As may be observed from these expressions, the finite difference zone with the lowest $v_{s}$, for a given $\Delta l$, will limit the highest frequency that can pass through the zone without numerical distortion. For the finite difference analyses performed in this investigation, $0.3 \mathrm{~m}$ by $0.3 \mathrm{~m}$ zones were used in subgrids one and two (refer to Figure 3). The top layer of the backfill has the lowest $v_{s}$ (i.e., $160 \mathrm{~m} / \mathrm{second}$ ). Using Equation (14b) and $\Delta l=0.3 \mathrm{~m}$, the finite difference grid used in the analyses should adequately propagate shear waves having frequencies up to approximately $53 \mathrm{~Hz}$. This value is well above the $15 \mathrm{~Hz}$ cutoff frequency used in the SHAKE analysis to compute the input motion for the finite difference analyses and well above the estimated fundamental frequency of the retaining wall-soil system being modeled (i.e., $\sim 6 \mathrm{~Hz}$ ).

\subsection{Damping}

An elasto-plastic constitutive model, in conjunction with the Mohr-Coulomb yield criterion, was used to model the soil, which inherently introduces the following hysteretic damping:

$$
D=\frac{2}{\pi} \cdot\left[1-\frac{G}{G_{\max }}\right]
$$

where $D$ is the hysteretic damping; $G$ is secant shear modulus; and $G_{\max }$ is small strain, or preyield, $G$. As may be observed from this expression, once the induced dynamic shear stresses exceed the shear strength of the soil, the plastic deformation of the soil introduces hysteretic damping, up to $D=2 / \pi$ at large strains. However, for dynamic shear stresses less than the shear strength of the soil (i.e., $G=G_{\max }$ ), the soil behaves elastically (i.e., $D=0)$.

As discussed in detail by $\mathrm{Ni}$ (2007), the sudden change in $G$ at yielding and at the stress reversals cause a numerical distortion that results in a "migrating" stress-strain response. To minimize this distortion, it is common to apply additional damping to the model, typically $2 \%$ to $3 \%$ (e.g., Finn, 1988; Wang and Makdisi 1999). For the analyses performed, $3 \%$ additional Rayleigh damping was applied, where Rayleigh damping provides a relatively constant level of damping over a restricted range of frequencies. The central frequency corresponding to the specified damping ratio is typically set to either the fundamental period (small strain) of the system being modeled (an inherent property of the wall-soil system) or predominant period of the system response (an inherent property of the wall-soil system and the ground motion). For the finite difference analyses performed, the central frequency was set equal to the small strain fundamental frequency of the retaining wall-soil system (i.e., $\sim 6 \mathrm{~Hz}$ ).

\section{COMPARISON OF FINITE DIFFERENCE AND SIMPLIFIED ANALYSES}

A series of analyses were performed using the model of the wall-soil system described above, scaling the input motions to different peak ground acceleration values. Each run required between 25 and 35 days to complete (long-run times is one of the shortcomings of explicit analyses). To assess the applicability of simplified techniques that were developed for estimating dynamic earth pressures and permanent wall displacement of gravity retaining walls to cantilever walls, comparisons of the results from the finite difference and simplified analyses are presented below. The reader is referred to Ebeling and Morrison (1992), Green et al. (2003), and Green and Michalowski (2006) for more detailed discussions about the simplified techniques used.

\subsection{Dynamic earth pressures}

3.1.1 Simplified procedure for computing earth pressures. The Mononobe-Okabe method for determining seismically induced active and passive lateral earth pressures is based on limit equilibrium and is an extension of the Coulomb theory for static stress conditions. The method entails three fundamental assumptions (e.g., Seed and Whitman, 1970):

1. Wall movement is sufficient to ensure either active or passive conditions, as the case may be.

2. The driving soil wedge inducing the lateral earth pressures is formed by a planar failure surface starting at the heel of the wall and extending to the free surface of the backfill. Along this failure plane the maximum shear strength of the backfill is mobilized.

3. The driving soil wedge and the retaining structure act as rigid bodies and, therefore, experience uniform accelerations throughout the respective bodies.

As demonstrated by Dr. Ignacio Arango (Seed and Whitman, 1970), the dynamic earth pressures may be determined from analogous static conditions. Accordingly, the Mononobe-Okabe expressions for dynamic earth pressures can be derived from Coulomb's expressions for static earth pressures. The analogous static conditions are achieved by rotating the wall-backfill system by an angle $\psi$, such that the vector sum of the horizontal and vertical inertial coefficients ( $k_{h}$ and $k_{v}$, respectively) is oriented vertically, where $\tan (\psi)=k_{h} /\left(1-k_{v}\right)$. In regards to the mathematical expressions, the MononobeOkabe expressions can be derived from Coulomb's expressions by replacing the static values for the total unit weight of the soil $\left(\gamma_{t}\right)$, height of the wall $(H)$, inclination of the backfill $(\beta)$, and inclination of the wall face from 
the vertical $(\theta)$, with the corresponding dynamic values (i.e., $\gamma_{t d}, H_{d}, \beta_{d}$, and $\theta_{d}$ ). This substitution results in the following set of equations for active conditions (i.e., $k_{h}$ acting away from the backfill):

$$
\begin{gathered}
P_{A E}=\frac{1}{2} \cdot \gamma_{t d} \cdot H_{d}^{2} \cdot K_{A}\left(\beta_{d}, \theta_{d}\right) \\
=\frac{1}{2} \cdot \gamma_{t} \cdot H^{2} \cdot\left(1-k_{v}\right) \cdot K_{A E} \\
K_{A E}=\frac{\cos ^{2}(\phi-\theta-\psi)}{\cos (\psi) \cdot \cos ^{2}(\theta) \cdot \cos (\delta+\theta+\psi)} \\
\cdot \frac{1}{\left[1+\sqrt{\left.\frac{\sin (\phi+\delta) \cdot \sin (\phi-\beta-\psi)}{\cos (\delta+\theta+\psi) \cdot \cos (\beta-\theta)}\right]^{2}}\right.}
\end{gathered}
$$

where, $P_{A E}$ is the resultant force acting on the wall.

A plot of the Mononobe-Okabe active earth pressure coefficients (i.e., $K_{A E}$ ) as a function of the horizontal inertial coefficient $\left(k_{h}\right)$ is shown in Figure 8a for $\beta=\delta=$ $\theta=0^{\circ}$ and $\phi^{\prime}=35^{\circ}$. As shown in this figure, when $k_{h}=$ 0 (i.e., static conditions), the value of $K_{A E}$ is equivalent
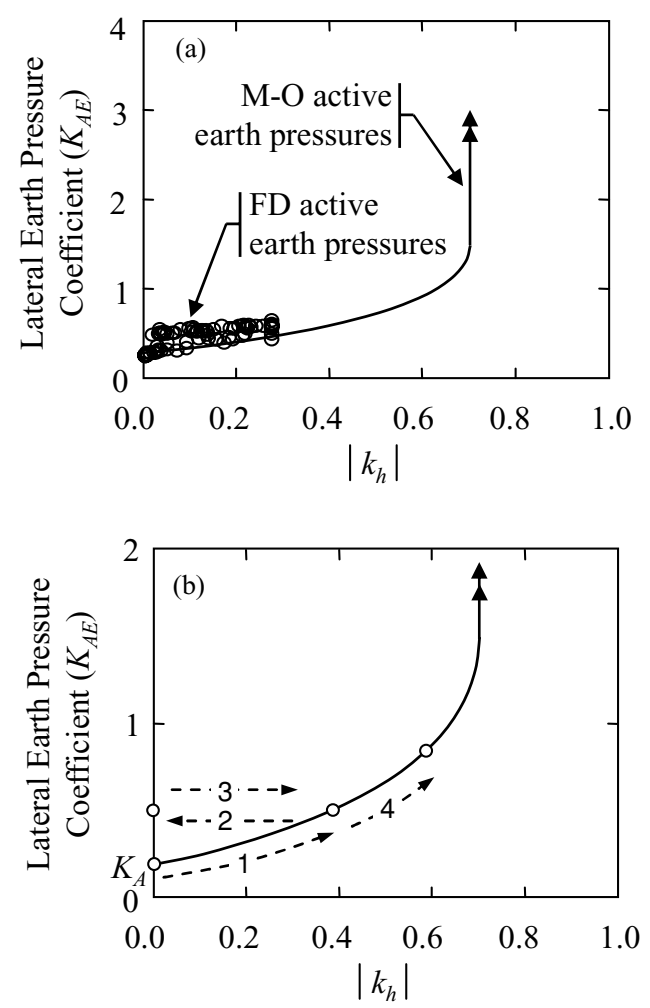

Fig. 8. Lateral earth pressure coefficients: (a) Mononobe-Okabe (M-O) and finite difference (FD) lateral earth pressure coefficients for $\beta=\theta=\delta=0^{\circ}$ and $\phi^{\prime}=35^{\circ}$; and (b) Illustration of the process by which lateral stresses are "locked-in." to Coulomb's active coefficients $\left(K_{A}\right)$. However, as $k_{h}$ increases in value, $K_{A E}$ becomes greater than $K_{A}$. As $k_{h}$ increases, the analogous static condition is achieved by tilting the wall forward, thus increasing the inclination of the backfill and increasing the pressure induced on the wall. The limiting pressure occurs when for the analogous static condition, the inclination of the backfill equals the angle of internal friction (i.e., $\beta_{d}=\phi^{\prime}$ ). At this point, the failure wedge becomes infinite in size, or synonymously, the angle of the failure plane $\left(\alpha_{A E}\right)$ from the horizontal equals the static inclination of the backfill (i.e., $\alpha_{A E}=\beta$, where $\alpha_{A E}$ decreases as $k_{h}$ increases). For such a case, no sized wall could restrain the backfill from movement.

3.1.2 Finite difference computed earth pressures on the cantilever wall. The dynamically induced lateral earth pressures acting on the stem of the wall were computed using finite difference. The corresponding lateral earth pressure coefficients $\left(K_{F D}\right)$ were computed from these stresses using the following expression (Green et al., 2003):

$$
K_{F D}=\frac{2 \cdot P_{F D}}{\gamma_{t} \cdot H^{2} \cdot\left(1-k_{v}\right)}
$$

where $P_{F D}$ is the resultant of the finite difference computed stresses acting on the stem of the wall; $\gamma_{t}$ is the total unit weight of the backfill; $H$ is the height of the wall; and $k_{v}$ is the vertical inertial coefficient (assumed to be zero). Equation (16) inherently assumes that the lateral stress distribution is triangular, with the base of the triangle at the depth of the base of the wall. This assumption is consistent with that used in the derivation of the Mononobe-Okabe expressions (i.e., Equation (15a)). Equation (16) was used to compute $K_{F D}$ values at times corresponding to the peaks in the time history of the horizontal inertial coefficient $\left(k_{h}\right)$ acting away from the backfill (i.e., active-type conditions), wherein spurious high-frequency spikes were filtered from the $k_{h}$ time history ( $\mathrm{Ni}, 2007)$.

A plot of the computed $K_{F D}$ values versus $k_{h}$ is also shown in Figure 8a, labeled FD active earth pressures, for an analysis in which a motion recorded during the $1989 M_{w} 6.9$ Loma Prieta earthquake in California was used. The Mononobe-Okabe lateral dynamic earth pressure coefficient $\left(K_{A E}\right)$ for the wall-soil system discussed above is also shown in this figure. The reason for the deviation of the finite difference-computed stresses and those computed by the Mononobe-Okabe expressions can be understood from examining Figure 9. Shown in this figure is the deformed mesh from one of the finite difference analyses, wherein the deformations are magnified by a factor of 3. At large values of $k_{h}$ directed away from the backfill, the induced inertial forces on the structural 


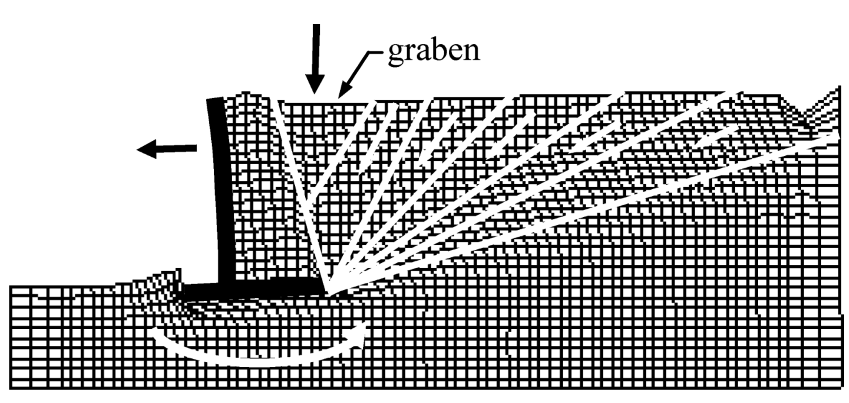

Fig. 9. Annotated deformed mesh from one of the finite difference analyses; deformations magnified by a factor of 3 .

Note: Toe of wall not initially embedded. The deformed mesh shown in this figure is only for illustration purposes. It is from an analysis wherein the acceleration time history was scaled to a very large peak acceleration. For this analysis, the right lateral boundary should have been farther from the wall.

block cause it to simultaneously bend, rotate, and potentially slide away from the backfill, at which time a small wedge of soil or graben moves vertically downward. (The structural block consists of the cantilever wall and the backfill contained within; see Figure 2.) As the direction of $k_{h}$ reverses (i.e., changes direction from away to toward the backfill), the graben prevents the structural block from returning to its undeformed shape, in effect locking in the elastic stresses resulting from the bending and rotation of the structural block.

This process is illustrated by the dashed arrows and corresponding earth pressure coefficients in Figure 8b, wherein the initial stresses imposed on the stem of the wall correspond to active conditions. As $k_{h}$ increases in the direction away from the backfill, the stresses on the stem increase according to the Mononobe-Okabe expression for active conditions (arrow 1). However, upon reversal of the direction of $k_{h}$, the stresses imposed on the stem do not decrease as predicted by the Mononobe-Okabe expression, but rather remain relatively constant (arrow 2). As the direction of $k_{h}$ reverses again in the direction away from the backfill, the stresses acting on the stem remain relatively constant until $k_{h}$ reaches the Mononobe-Okabe "envelope" (arrow 3), at which point the stresses increase again according to the Mononobe-Okabe expression for active conditions (arrow 4). This stepwise increase in the "locked-in" stresses continues until the residual stresses imposed on the stem correspond to at-rest (or $K_{o}$ ) conditions, while the dynamically induced inertial stresses are superimposed on the locked-in residual stresses. The increase in residual stresses is clearly shown in Figure 10, wherein plots are shown of both the time history of $k_{h}$ and of $P_{F D}$.

The locked-in residual stresses on the wall are not released by the slippage of the wall away from the backfill. This is because the "driving soil wedge" is not monolithic, but rather, in this case, consists of a graben and five

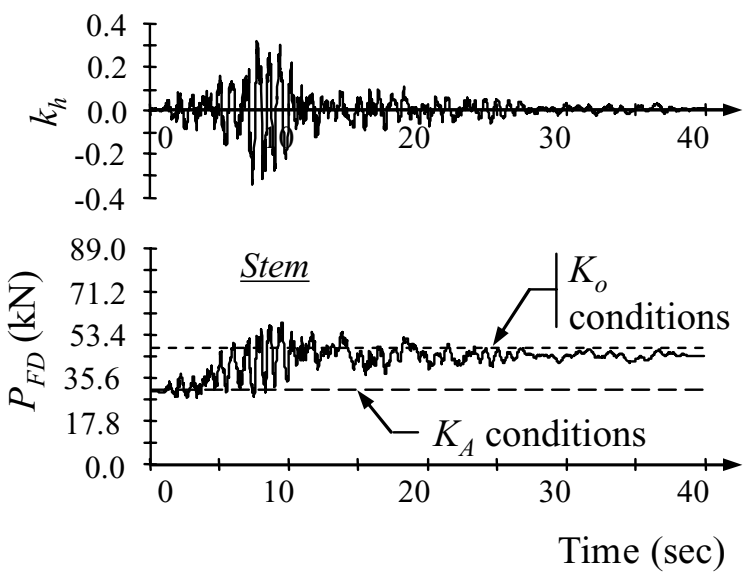

Fig. 10. Time history of the horizontal inertial coefficient $\left(k_{h}\right)$ at approximately the center of the structural block, and the time history of the resultant of the imposed stresses $\left(P_{F D}\right)$ on the stem of the cantilever retaining wall.

driving soil wedges (Figure 9), with the latter tending to move downward and away from the backfill as the wall slides outward. As a result, the graben "rides along" with the driving soil wedges maintaining its role of locking in the residual stresses. The progressive increase in residual stresses was also observed in centrifuge model tests performed by Andersen et al. (1991), as shown in Figure 11. Further validating the multidriving soil wedge response are the shaking table test results on a model retaining (Aitken, 1982). A post-test photo is shown in Figure 12a; for scale, the height of the retaining wall shown in this photo is $0.32 \mathrm{~m}$. The model was subjected to horizontal base excitations that were generated by a lever armspring release mechanism. The excitations were decaying sine waves (Figure 12b), in which the first two to three cycles exceeded acceleration required to cause sliding. As may be observed from Figure 12a, two driving wedges

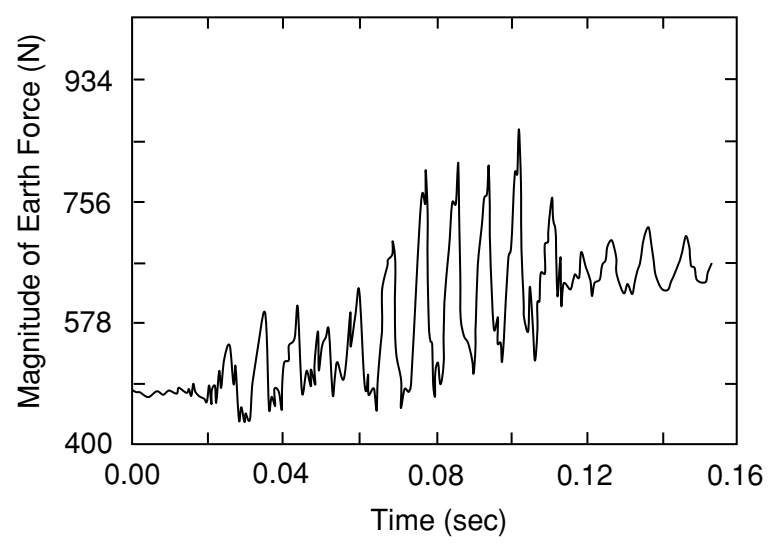

Fig. 11. Lateral force induced on retaining wall by backfill in centrifuge model tests (adapted from Andersen et al., 1991). 


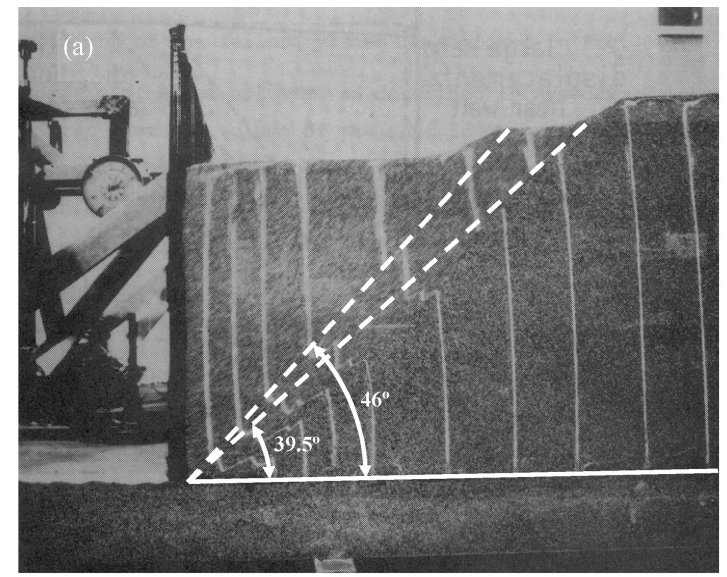

(b)

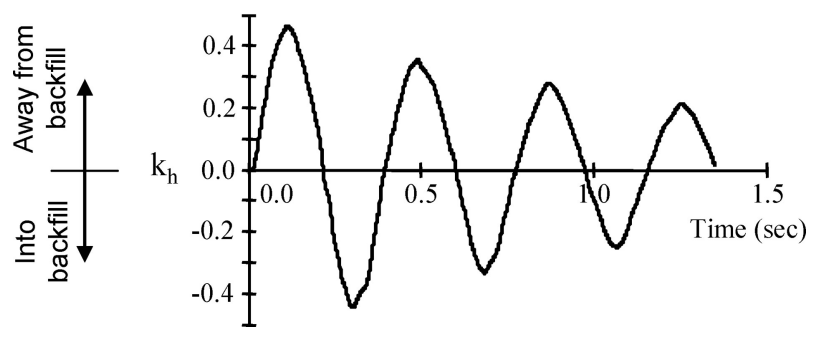

Fig. 12. Shaking table test results on a model retaining wall: (a) A posttest photograph showing two predominant driving failure wedges; and (b) Excitation time history. (Adapted from Aitken, 1982).

fully developed in the backfill, with the slip planes inclined at $\sim 39.5^{\circ}$ and $\sim 46^{\circ}$ resulting from the first and second active pulses in the excitation, respectively.

\subsection{Permanent wall displacement}

Comparisons of the permanent relative displacements $\left(d_{r}\right)$ of the wall computed by finite difference and Newmark sliding block analyses (Newmark, 1965) of the structural block (Figure 2) are shown in Figure 13. From the finite difference analyses, $d_{r}$ was computed by subtracting the total displacement of the structural node at the intersection of the stem and base of the wall from the total displacement of the grid point at the free-field boundary at the same depth. As may be observed from Figure 13, $d_{r}$ computed from the finite difference analyses is about $0.33 \mathrm{~m}$.

Newmark sliding block analyses of the structural block were performed using the acceleration time history shown in Figure 14. This time history was computed in the finite difference analyses at the free-field boundary at a depth corresponding to approximately mid-height of the structural block. To perform a Newmark sliding block
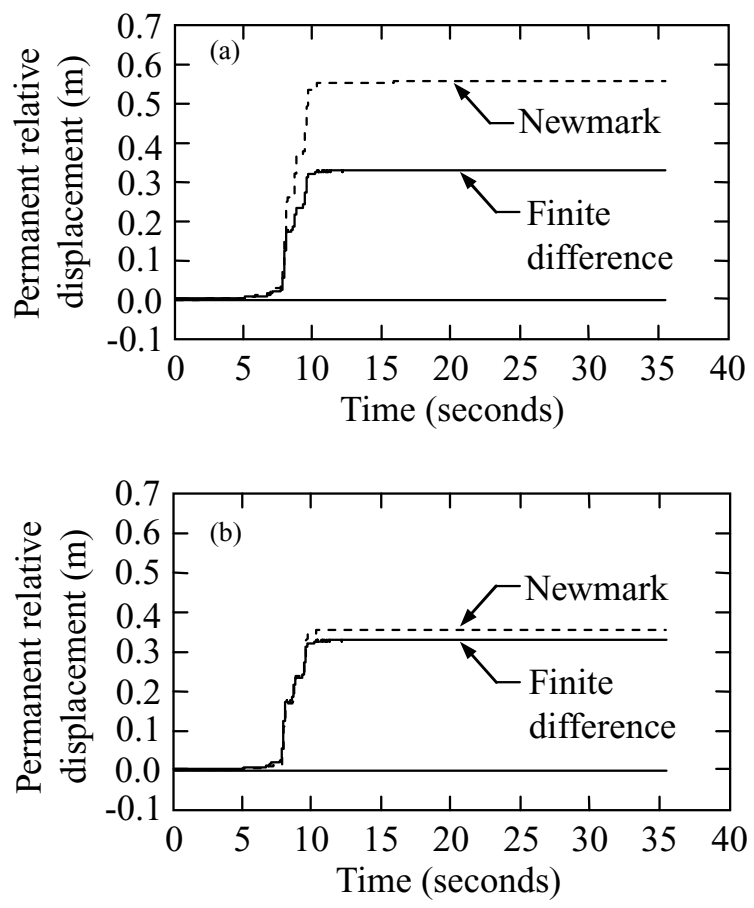

Fig. 13. Comparison of the permanent relative displacements computed by finite difference and Newmark sliding block analyses (Newmark, 1965): (a) Permanent relative displacements computed via the Newmark sliding block procedure assuming $N^{*} \cdot g=0.22 \mathrm{~g}$; and (b) Permanent relative displacements computed via the Newmark sliding block procedure assuming $N^{*} \cdot g=0.27 \mathrm{~g}$.

analysis, a maximum transmissible acceleration $\left(N^{*} \cdot g\right)$ has to be specified, which is the value of acceleration imparted to the block resulting in a factor of safety against sliding equal to 1.0. Using the interface friction angle between the concrete wall and foundation soil (i.e., $\delta=31^{\circ}$ ) in conjunction with the weight of the structural block, $N^{*} \cdot g$ was determined to be approximately $0.22 \mathrm{~g}$. The sliding block analysis resulted in $d_{r}=0.55 \mathrm{~m}$, as shown in Figure 13a, which is considerably larger than that from the finite difference analysis.

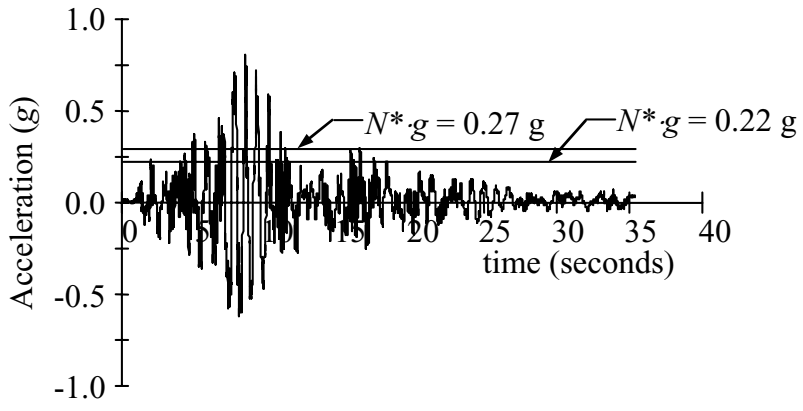

Fig. 14. Acceleration time history used in the Newmark sliding block analysis of the structural block. 
A possible explanation for the difference in the $d_{r}$ values is that the sliding block analysis did not account for additional sliding resistance resulting from the "plowing action" that occurs at the toe of the wall. Although the wall was not embedded in the foundation soil in its initial, undeformed shape, the wall tended to rotate around the toe as it translated away from the backfill. As a result, the toe of the wall penetrated and plowed through the foundation soil. Such a mechanism was observed in the deformed finite difference mesh (Figure 9). To account for this additional resistance to sliding, $N^{*} \cdot g$ was recomputed assuming a friction angle of $35^{\circ}$, which is between the interface friction angle (i.e., $\delta=31^{\circ}$ ) and the $\phi^{\prime}$ of the foundation soil (i.e., $40^{\circ}$ ), with the revised value of $N^{*} \cdot g=0.27 \mathrm{~g}$ (Figure 14). A comparison of the permanent relative displacements computed from the finite difference and the sliding block analyses using the revised value of $N^{*} \cdot g$ is shown in Figure 13b. As may be observed from this figure, the predicted displacements are in very close agreement.

\section{ADDITIONAL OBSERVATIONS}

From an engineering perspective, the main concern for a gravity retaining wall system is the global stability of the wall (i.e., sliding, overturning, and bearing capacity failure). The structural design of such walls is a secondary issue because the walls are so massive and have a relatively small aspect ratio in cross section. However, this is not the case for cantilever walls, for which proper structural design is just as important as global stability. Consequently, the critical load cases for structural design and global stability need to be determined in designing a cantilever retaining wall system. A common assumption in practice is that the critical load case for global stability, which is often determined using the Mononobe-Okabe procedure, is also the critical load case for structural design. However, the finite difference analyses showed that this assumption is not valid.

Figure 15 illustrates the critical load cases for both structural design and global stability from one of the finite difference analyses. As shown in Figures 15a and 15c, the critical load case for global stability occurs when the horizontal ground acceleration is directed into the backfill (or correspondingly, when $k_{h}$ is directed away from the backfill). For this case, in essence, the structural block is treated as if it were a gravity retaining wall. Accordingly, the stresses and resultant force acting on the interface of the structural block and driving soil wedge (i.e., the vertical section through the heel of the wall) are of interest. As may be observed from Figure 15a, the stresses acting on the structural block are relatively triangularly distributed, similar to the stress distribution assumed for
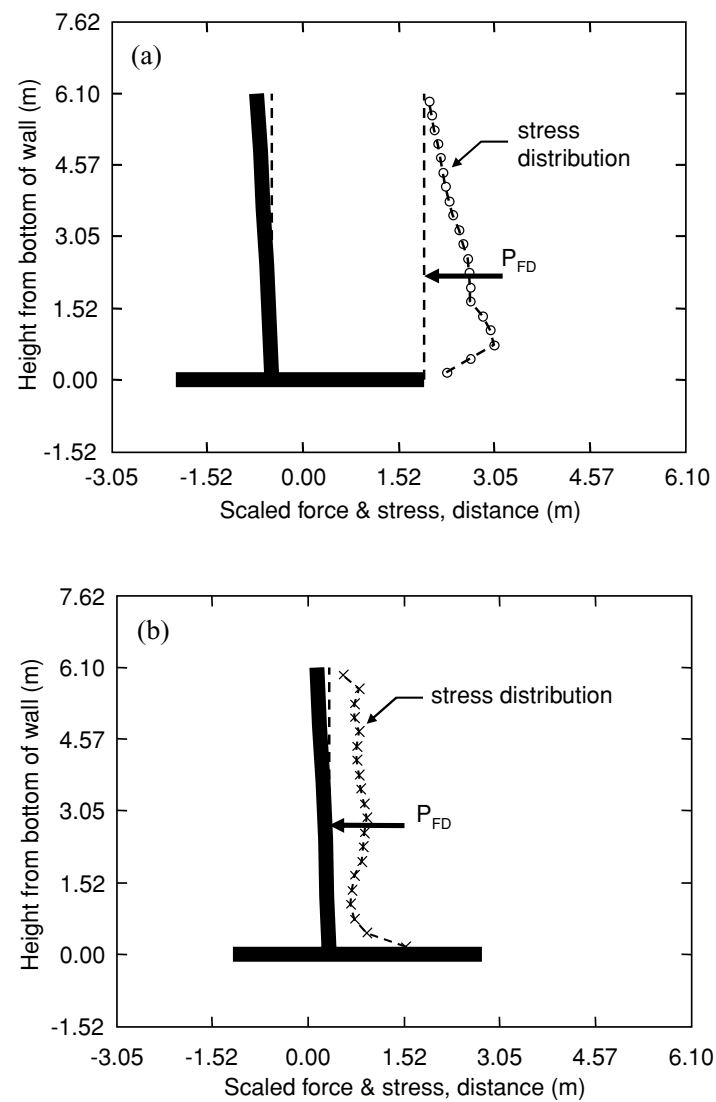

(c)

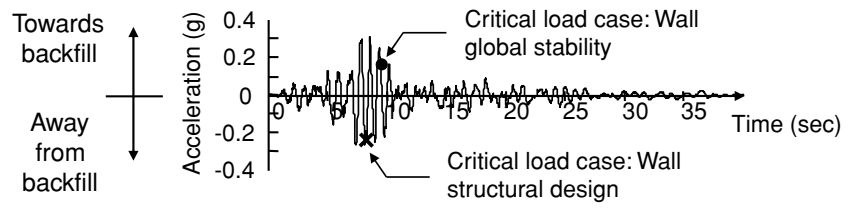

Fig. 15. Critical load cases: (a) for global stability of the wall; and (b) for structural design. (c) Free-field, free surface acceleration time history computed at the elevation of the wall base. Denoted in this time history are the accelerations and times corresponding to the critical load cases for both the structural design and global stability.

static analyses of walls (Note: In Figure 15a, the reduction in stress toward the base of the wall is due to the arching of the soil in the failure wedge from soil below the failure plane to the structural block.). As a result, the point of action of the resultant force is around one-third the height of the wall from the base.

In contrast to the critical load case for global stability, the critical case for structural design is shown in Figures $15 \mathrm{~b}$ and $15 \mathrm{c}$. For this case, the stresses and resultant force acting directly on the stem of the wall are of interest. As may be observed from Figure 15c, this case occurs when the ground acceleration is directed away from the backfill (or correspondingly, when $k_{h}$ is directed into the 
backfill). As a result, the stresses imposed on the wall by the soil are relatively uniformly distributed, and the point of action of the resultant force is about mid-height of the wall.

Although the magnitudes of the respective resultant forces are approximately the same for the finite difference results shown in Figures 15a and 15b (Note: This may not be the case for all earthquake motions.), it is the difference in the point of action of the resultant forces that distinguishes the critical load cases. Critical for structural design is the moment induced at the bottom of the stem. Hence, for a given resultant force, the higher the point of action above the base of the wall, the larger the induced moment around the bottom of the stem. Consequently, in all the analyses performed by the authors, the critical load cases for structural design occurred when $k_{h}$ was directed toward the backfill (and hence, the soil imposed a fairly uniform stress along the height of the stem) and the point of action of the resultant force was approximately mid-height of the wall.

\section{SUMMARY AND CONCLUSIONS}

The authors outline the details of a system model and its calibration and validation for use in computing the dynamic response of a cantilever retaining wall. The soil was modeled using an elastic-perfectly plastic constitutive relation, with the Mohr-Coulomb envelope defining the yield criterion. The plastic flow rule was assumed to be nonassociative. The wall was modeled with elastic beam elements using a cracked second moment of area ( $\left.I_{\text {cracked }}\right)$ equal to $0.4 \times I_{\text {uncracked }}$. Interface elements are used to model the wall-soil interface, wherein the interface element parameters are those that give a best fit of the Gomez et al. (2000a, b) hyperbolic interface model. The lateral stresses induced on the wall computed in the finite difference analyses did not correspond with those predicted by the Mononobe-Okabe method. The reason for this deviation is attributed to the relative flexibility of the structural block and to the nonmonolithic motion of the driving soil wedge, both of which violate assumptions inherent in the Mononobe-Okabe method. The permanent relative displacement of the wall computed in the finite difference analyses were in general accord with those predicted using the Newmark sliding block procedure, once the plowing action at the toe was taken into account. Finally, it was found that the critical load case of structural design of the wall differed from that for global stability, which is contrary to the common assumption made in practice that the two load cases are the same. Specifically, the critical load case for global stability occurs when $k_{h}$ is directed away from the backfill and the critical case for structural design occurs when $k_{h}$ is directed toward the backfill.

\section{ACKNOWLEDGMENTS}

A portion of this study was funded by the Headquarters, U.S. Army Corps of Engineers (HQUSACE) Civil Works Earthquake Engineering Research Program (EQEN). Dr. Robert M. Ebeling was the cognizant Corps representative; his suggestions and comments throughout the project are greatly appreciated. The authors also acknowledge Itasca Consulting Group, Inc. for the academic loan of their FLAC code. This collaboration is very much appreciated.

\section{REFERENCES}

Aitken, G. H. (1982), Seismic Response of Retaining Walls, MS Thesis, University of Canterbury, Christchurch, New Zealand.

Andersen, G. A., Whitman, R. V. \& Germaine, J. T. (1991), Seismic response of rigid tilting walls, in Proceedings of Centrifuge '91, Balkema, Rotterdam, The Netherlands, pp. 41724.

Ebeling, R. M. \& Filz, G. M. (2001), Soil-Structure Interaction Analysis of Rock-founded Cantilever and Mass Concrete Retaining Walls, Draft Report, US Army Corps of Engineers, Engineer Research and Development Center, Vicksburg, MS.

Ebeling, R. M. \& Morrison, E. E. (1992), The Seismic Design of Waterfront Retaining Structures, US Army Technical Report ITL-92-11, US Navy Technical Report NCEL TR-939, US Army Engineer Waterways Experiment Station, Vicksburg, MS.

Finn, W. D. L. (1988), Dynamic analyses in geotechnical engineering, in J. L. Von Thun (ed.), Earthquake Engineering and Soil Dynamics II-Recent Advances in Ground-Motion Evaluation, Geotechnical Special Publication 20, ASCE, pp. 523-91.

Gomez, J. E., Filz, G. M. \& Ebeling, R. M. (2000a), Extended Load/Unload/Reload Hyperbolic Model for Interfaces: Parameter Values and Model Performance for the Contact between Concrete and Coarse Sand, ERDC/ITL TR-00-7, US Army Corps of Engineers, Engineer Research and Development Center.

Gomez, J. E., Filz, G. M. \& Ebeling, R. M. (2000b), Development of an Improved Numerical Model for Concrete-to-Soil Interfaces in Soil-Structure Interaction Analyses, Report 2, Final Study, ERDC/ITL TR-99-1, US Army Corps of Engineers, Engineer Research and Development Center.

Green, R. A. \& Ebeling, R. M. (2002), Seismic Analysis of Cantilever Retaining Walls, Phase 1, ERDC/ITL TR-02-3, US Army Corps of Engineers, Engineer Research and Development Center, http://www-personal.umich.edu/ rugreen/ papers/ITL-TR-02-3.pdf.

Green, R. A. \& Michalowski, R. L. (2006), Shear band formation behind retaining structures subjected to seismic excitation, Foundations of Civil and Environmental Engineering, 7, 157-69.

Green, R. A., Olgun, C. G., Ebeling, R. M. \& Cameron, W. I. (2003), Seismically induced lateral earth pressures on a cantilever retaining wall, in J. E. Beavers (ed.), Advancing Mitigation Technologies and Disaster Response for Lifeline Systems, ASCE Technical Council on Lifeline Earthquake Engineering Monograph No. 25, pp. 946-55. 
Headquarters, US Army Corps of Engineers. (1989), Retaining and Flood Walls, EM 1110-2-2502, Washington DC.

Headquarters, US Army Corps of Engineers. (1992), Strength Design for Reinforced-Concrete Hydraulic Structures, EM 1110-2-2104, Washington DC.

Idriss, I. M. \& Sun, J. I. (1992), User's Manual for SHAKE91: A Computer Program for Conducting Equivalent Linear Seismic Response Analyses of Horizontally Layered Soil Deposits, Center for Geotechnical Modeling, Department of Civil and Environmental Engineering, University of California, Davis, CA.

Itasca. (2000), FLAC (Fast Lagrangian Analysis of Continua) User's Manuals, Itasca Consulting Group, Minneapolis, MN.

Jaky, J. (1944), The coefficient of earth pressure at rest, Magyar Menok es Epitesz Kozloi (Journal of the Society of Hungarian Architects and Engineers), October, 355-358.

Kuhlemeyer, R. L. \& Lysmer, J. (1973), Finite element method accuracy for wave propagation problems, Journal of the Soil Mechanics and Foundations Division, 99(SM5), 42127.

Lysmer, J., Udaka, T., Tsai, C.-F. \& Seed, H. B. (1975), FLUSH: A Computer Program for Approximate 3-D Analysis of SoilStructure Interaction Problems, EERC Report No. EERC75-30, Earthquake Engineering Research Center, University of California, Berkeley, CA.

MacGregor, J. G. (1992), Reinforced Concrete Mechanics and Design, Prentice Hall, Englewood Cliffs, NJ.

Mononobe, N. \& Matsuo, H. (1929), On the determination of earth pressures during earthquakes, in Proceedings: World Engineering Congress, 9, 177-85.

Nadim, F. \& Whitman, R. V. (1983), Seismically induced movements of retaining walls, Journal of Geotechnical Engineering, ASCE, 109(7), 915-31.

Newmark, N. M. (1965), Effects of earthquakes on dams and embankments, Geotechnique, 15(2), 139-60.

$\mathrm{Ni}$, B. (2007), Implementation of a bubble model in FLAC and its application in dynamic analysis, Ph.D. thesis, University of Auckland, Auckland, New Zealand.
Okabe, S. (1924), General theory of earth pressures, Journal of Japan Society of Civil Engineering, 10(6), 1277-323, plus figures.

Ostadan, F. (2005), Seismic soil pressure for building walls: An updated approach, Soil Dynamics and Earthquake Engineering, 25, 785-93.

Paulay, T. \& Priestley, M. J. N. (1992), Seismic Design of Reinforced Concrete and Masonry Buildings, John Wiley and Sons, New York, NY.

Richards, R., Jr. \& Elms, D. G. (1979), Seismic behavior of gravity retaining walls, Journal of Geotechnical Engineering, ASCE, 105(4), 449-69.

Seed, H. B. \& Whitman, R. V. (1970), Design of Earth Retaining Structures for Dynamic Loads, Lateral Stresses in the Ground and Design of Earth-Retaining Structures, ASCE, $103-47$.

Stokoe, K. H., II, Wright, S. G., Bay, J. A. \& Roesset, J. M. (1994), Characterization of geotechnical sites by SASW method, in R. D. Woods (ed.), Geophysical Characterization of Sites, Oxford and IBH Publishing, New Delhi, 15-25.

Terzaghi, K. (1943), Theoretical Soil Mechanics, John Wiley and Sons, New York, NY.

Veletsos, A. \& Younan, A. H. (1994), Dynamic soil pressure on rigid vertical walls, Earthquake Engineering and Structural Dynamics, 23, 275-301.

Wang, Z. L. \& Makdisi, F. I. (1999), Implementing a boundary surface hypoplasticity model for sand into FLAC program, in Detoumay \& Hart (eds.), FLAC and Numerical Modeling in Geomechanics, Balkema, Rotterdam, pp. 483-90.

Wood, J. H. (1973), Earthquake induced soil pressures on structures, Ph.D. thesis, EERL 73-05, California Institute of Technology, Pasadena, CA.

Woods, R. D. (1994), Borehole methods in shallow seismic exploration, in R. D. Woods (ed.), Geophysical Characterization of Sites, Oxford and IBH Publishing, New Delhi, pp. 91-100.

Wu, G. \& Finn, W. D. L. (1999), Seismic lateral pressures for design of rigid walls, Canadian Geotechnical Journal, 36, 50922 . 